Division of Geological \& Geophysical Surveys

PUBLIC-DATA FILE $93-47$

\title{
GEOLOGIC MAP OF SLEETMUTE C-7, D-7, C-8, AND D-8 QUADRANGLES, HORN MOUNTAINS AREA, SOUTHWEST ALASKA
}

by

T.K. Bundtzen, G.M. Laird, E.E. Harris, J.T. Kline, and M.L. Miller

July 1993

THIS REPORTS HAS NOT BREN REVIEWED FOR TECHNICAI CONTENT (BXCEPT AS NOTED IN TEXT) OR FOR CONFORMITY TO THE EDITORIAL STANDARDS OF DGGS.

\section{Released by}

STATB OF ALASKA

DEPARTMENT OP NATURAL RESOURCES

Division of Geological \& Geophysical Surveys

794 University Avenue, Suite 200

Fairbanks, Alaska 99709-3645 
Geology of the Sleetmute C-7, D-7, C-8, and D-8 Quadrangles, Horn Mountains, Alaska

By T.K. Bundtzen, G.M. Laird, E.E. Harris, J.T. Kline, and M.L. Miller

\section{INTRODUCTION AND GEOGRAPHY}

During the 1990, 1991, and 1992 field seasons, four 1:63,360 scale quadrangles were geologically mapped in the northwest corner of the Sleetmute Quadrangle of southwest Alaska (figure 1). The $2,220 \mathrm{~km} 2$ area consists of broad sediment-filled lowlands interdispersed with rounded ridgelines and small mountain massifs that range in elevation from 500 to 1.070 meters. Most of the streams and rivers in the study area drain into the Kuskokwim River, which flows through the southem portion of the map area and is one of Alaska's largest river systems. However, the Iditarod River flows northeastward through the Sleetmute D-8 quadrangle eventually reaching the Innoko and Yukon River drainage basins. Although much of the study area has not been glaciated, the rugged Horn Mountains and adjacent lowlands has experienced at least four separate glaciations.

Most of the field work was conducted from several spike camps in various locations within the study area; heliocopter support was utilized in remote areas west and south of the Kolmokof and Iditarod Rivers.

The preliminary results presented in this report consist of: 1) a brief introductory text; 2) seven tables that depict paleocurrent data (table 1), major oxide and trace element analyses of igneous rocks (tables 2 and 3), 40Ar-39Ar and $\mathrm{K} 40-\mathrm{Ar} 40$ isotopic age determinations (tables 4 and 5), C14-C13 age determinations (table 6 ), and trace element analyses of mineralized zones (table 7); and 3) the geologic map itself accompanied by complete map unit descriptions (plate 1).

\section{GEOLOGIC SUMMARY}

Geologic units in the study area are subdivided into four basic assembleges: 1) Cretaceous flysch; 2) Upper Cretaceous mafic to felsic volcanic rocks; 3) Upper Cretaceous and Lower Tertiary(?) intermediate to felsic plutonic rocks; and 4) widespread unconsolidated lithologies that range in age from Late Tertiary through the Quaternary. During discussion of these geologic units, 


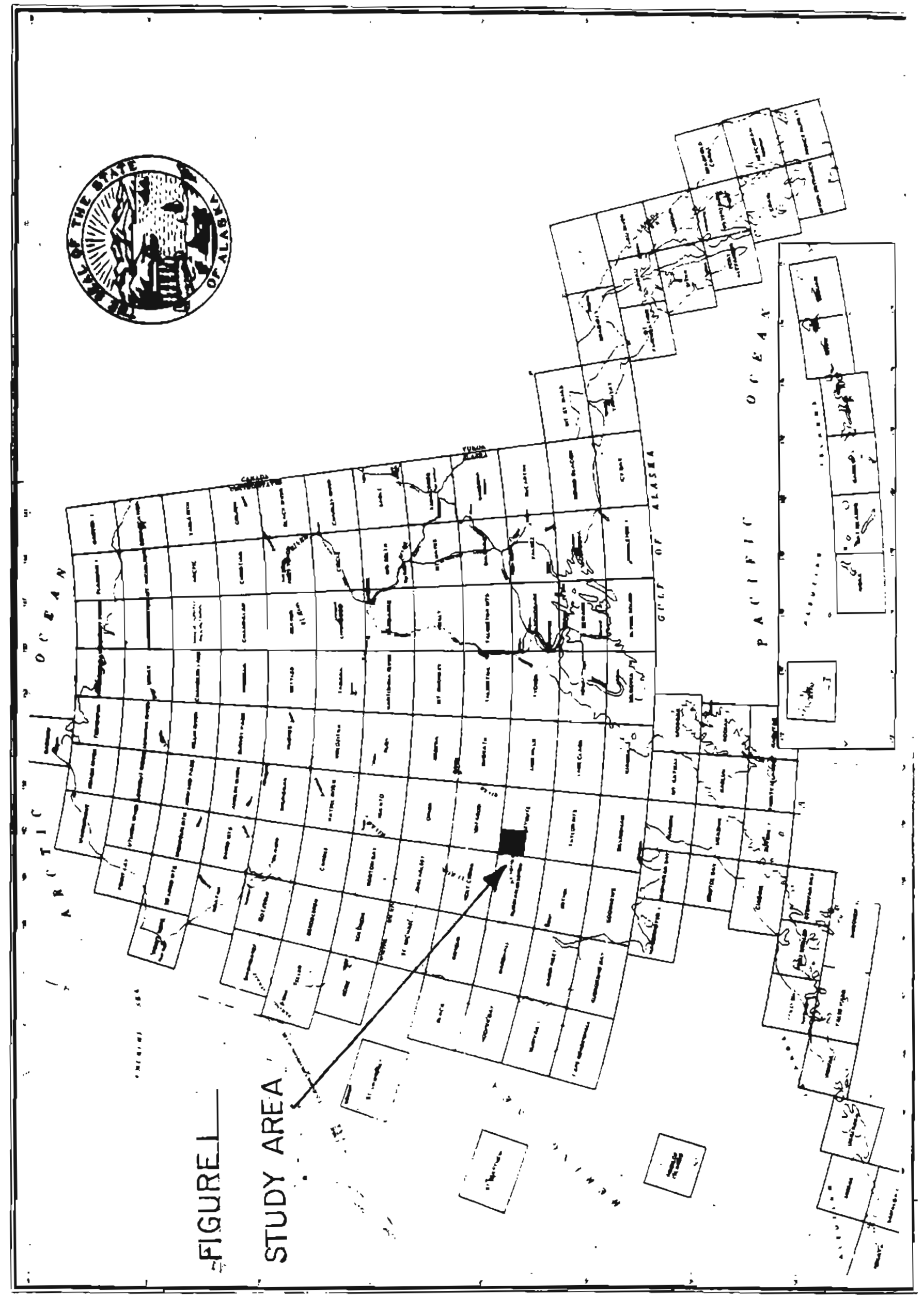


the reader is referred to the geologic map (plate 1) for more complete descriptions.

Kuskokwim Group

The major stratigraphic units exposed in the study area belong to the Kuskokwim Group, after Cady and others (1955), which consists of sandstone, shale, siltstone, and conglomerate. The Kuskokwim Group has been subdivided onto eight lithologic units (see plate 1) that we have interpreted to represent turbidite fan, foreslope, shallow-marine, and possibly shoreline or nonmarine environments deposited in a marine regression during evolution of the sedimentary basin. Paleocurrent data (table 1) suggests southerly or southeasterly directions of sediment transport, which is consistant with data and interpretations presented by Miller and Bundtzen (1993) from the Kuskokwim Group in the Iditarod Quadrangle. Fossils collected during our work and summarized by Miller and Bundtzen (1993) for collections in the nearby Iditarod Quadrangle range in age from Cenomanian to Turonian (early-Late Cretaceous); however the top of the section is poorly constrained.

No total thickness was estimated during our work. We have discovered that the Cretaceous section is probably complexly repeated by open to recumbant folds, and perhaps thrust faults. Cady and others (1955) estimate that the Kuskokwim Group is at least 12,000 meters thick along the Kuskokwim River from Crooked Creek to Chuathbaluk; Bundtzen and Laird (1991), Bundtzen and others (1992), and Miller and Bundtzen (1993) estimate thicknesses from 2,000 to 5,000 meters for equivalent rocks northeast of the study area.

\section{Iditarod Volcanics}

Basaltic andesite, andesite to dacite tuff and agglomerate of the Iditarod Volcanics, after Miller and Bundtzen (1988), overlie the Kuskokwim Group in the northwest part of the map area. Three units were subdivided during our work (plate 1). Altered dacite tuffs and agglomerate are overlain by more mafic basaltic andesites; the total thickness is estimated at 350 meters in the study area. Miller and Bundtzen (1988) report that the basal units range in age from 75 to $77 \mathrm{ma}$, and the upper more mafic volcanics appear to range in age from 58 to $62 \mathrm{ma}$. We have no age control for the Iditarod Volcanics in the study area.

Horn Mountains Volcanic Field 
A remarkably complete, volcanic pile ranging in composition from basaltic andesite to rhyolite covers a circular shaped region in the Horn Mountains in the east-central part of the map area (plate 1). Twelve units were subdivided during our work (plate 1). Based on aerial mapping, isotopic age, and stratigraphic relationships, we have determined a regular stratigraphic succession that spans about 1.5 million years, taking into account analytical error (plate 1; table 4). $40 \mathrm{Ar}-39 \mathrm{Ar}$ ages range from $70.78 \mathrm{ma}$ for vitric tuffs near the base to 68.09 from andesite tuff near the top of the section. The basal portion of the Horn Mountains Volcanic field consists of vitric welded (?) tuffs that are successive overlain by basaltic andesite and andesite flows, latite flows and tuff, and finally by more felsic tuffs and flows. We estimate a total thickness at about 800 meters.

The circular 'ring' structure exhibited by the volcanic field as well as local faulted contacts suggest that the Horn Mountains Volcanic Field may represent a collapsed caldera active during latest Cretaceous time.

\section{Intrusive Rocks and Hornfels}

Seven plutonic units and hornfels were mapped during our work (plate 1). Granodiorite, minor granite, and rare quartz syenite comprize the Horn Mountains pluton, which underlies and intrudes the Horn Mountains Volcanic Field in the Horn Mountains. Isotopic age control (68.64 ma from granodiorite, table 4), major oxide and trace element chemistry (table 2,3), and field relationships suggest that the plutonic rocks are more-or-less coeval with the volcanics, consistant with other 'volcanic-plutonic' complexes known in the Kuskokwim Mountains.

Unusual, peraluminous granite and alaskite porphyry sills, stocks and dikes intrude the Kuskokwim Group throughout the study area. Their peraluminous character and rare-earth element depletion trends distinquish them from the larger plutonic bodies; however, they appear to be roughly the same age as the former granodiorite intrusions (68.64 to $71.98 \mathrm{ma}$; tables 4,5 ).

\section{Unconsolidated Deposits}

A wide varieky of unconsolidated deposits ranging in age from Late Tertiary to Holocene cover about 55\% of the map area. We have subdivided these into 21 alluvial, glacial, colluvial, and elluvial units (plate 1). These include aerially extensive glacio-fluvial deposits and till in the Horn Mountains and adjacent areas, where evidence for at least four separate glaciations has been recognized. A $40 \mathrm{Ar}-39 \mathrm{Ar}$ isotopic age on volcanic ash interbedded with old glacial outwash in 
the Qof unit was determined to be at least one million years old (unpublished data), suggesting that the oldest glacial events are midto-lower Pleistocene in age. However, we need more sample material to verify or refute this age. The youngest age on till in Getmuna Creek valley is 13,600 yrs bp (no. $7 \mathrm{~b}$, table 7 ), which is equivalent to till of Elmendorf age in the Mantanuska-Susitna River area near Anchorage (R.D. Reger, pers. comm., 1992).

Most unconsolidated deposits are Late Pleistocene or Holocene in age, and some stream alluvium is forming today.

\section{ECONOMIC GEOLOGY}

Gold polymetallic resources were investigated in the Horn Mountains, at Juninggulra Mountain, and at the Kolmokof and New York Creek mercury and placer gold mines respectively. In the Horn Mountains, greisen-like veins containing gold, silver, and base metals occur in both shallow, high-level portions of the Horn Mountains pluton, and in adjacent hornfels (map no. 15, 23, 24, 28, 30, 39, 47 . plate 1 ; table 7). Lode mercury deposits containing anomalous gold and tellurium are associated with altered mafic dikes at the Kolmokof Mine (53A,B; plate 1; table 7). Small deposits of cinnabar and stibnite occur in fissures and breccia zones within granite porphyry at Juninggulra Mountain north of the Horn Mountains (plate, 1, table 7).

Small placer deposits have been exploited intermittantly since 1910 at Murray Gulch, tributary to New York Creek, northeast of Napaimiut (plate 1; no. 63, table 7). Miller and others (1989) summarize the geology and history of development of selected mineral properties in the study area in more detail than is discussed here. Gray and others (in process) discusses favorable areas for metallic mineral resources in the Horn Mountains area using pan concentrate and stream sediment geochemistry.

Bundtzen and others (1989) discuss the availability of sand and gravel and riprap in the study area.

\section{ACKNOWLEDGEMENTS}

We wish to thank the late Bruce Hickok, Robert Rutherford, and June McAtee--all of Calista Corporation--for informative geologic discussions and for their encouragement and support to work on Calista lands in the study area. Paul Layer (University of AlaskaFairbanks Geophysical Institute) performed the 40Ar-39Ar isotopic age determinations on igneous rocks. Mitch Reynolds and Clementine 
Caudle-Wright--both with the U.S. Geological Survey-provided contract time extensions, and helpful advice during the contract period. This mapping project was funded, in part, by U.S. Geological Survey COGEOMAP grant no. 14-08-0001-A0875.

\section{REFERENCES CITED}

Bundtzen, T.K., and Laird, G.M., 1991, Geology and mineral resources of the Russian Mission C-1 Quadrangle, Alaska: Alaska Division of Geological and Geophysical Surveys Professional Report 109, 24 pages, two plates at 1:63,360 and 1:500 respectively.

Bundtzen,T.K., Laird, G.M., and Gilbert, W.G., 1989, Material Studies along Kuskokwim River, McGrath to Kalskag, Southwest Alaska: Alaska Division of Geological and Geophysical Surveys Public Data File Report 89-16, 76 pages.

Bundtzen, T.K, Miller, M.L., Laird, G.M., and Bull, K.F., 1992, Geology and mineral resources of the Iditarod Mining District, Iditarod B-4 and eastern B-5 Quadrangles, Alaska: Alaska Division of Geological and Geophysical Surveys Professional Report 97, 46 pages; two plates at $1: 63,360$ and $1: 1,000$ scales respectively.

Cady, W.M., Wallace, R.E., Hoare, J.M., and Webber, E.J., 1955, The central Kuskokwim Region, Alaska: U.S. Geological Survey Professional Paper 268, 132 pages, two plates at 1:400,000 scale.

Gray, J.E., Theodorakos, P.M., Bradley, L.A., and Bullock, J.H., in process, Favorable areas for metallic mineral resources in and near the Horn Mountains, Sleetmute Quadrangle, southwest Alaska: U.S. Geological Survey unpublished manuscript, 32 pages.

Miller, M.L., and Bundtzen T.K., 1988, Right lateral offset solution proposed for the Iditarod-Nixon Fork Fault, western Alaska: U.S. Geological Survey Circular 1016, p.99-103.

Miller, M.L., and Bundtzen, T.K., 1993, Generalized geologic map of the Iditarod Quadrangle showing potassium, major oxide, trace element, fossil, paleocurrent, and archeological sample localities: U.S. Geological Survey Miscellaneous Field Studies Map MF2219-A, 24 pages (est.), scale 1:250,000. 
Miller, M.L., Belkin, H.E., Blodgett, R.B., Bundtzen, T.K., Cady, J.W., Goldfarb, R.J., Gray, J.E., McGimsey, R.G., and Simpson, S.L., 1989, PreField study and mineral resource assessment of the Sleetmute Quadrangle, southwestern Alaska: U.S. Geological Survey Open File Report 89-363, 115 pages, 3 plates, scale 1:250,000. 


\begin{tabular}{|c|c|c|c|c|}
\hline 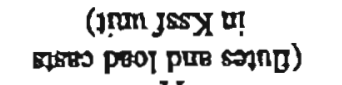 & & $\begin{array}{l}.80 I \\
.8 Z I\end{array}$ & & \\
\hline $\operatorname{mad}_{n}$ & هLII & $.81 I$ & I8Igl6 & 9 \\
\hline \multicolumn{5}{|l|}{ (j!̣m J58X } \\
\hline ㄸํ & &.$\$ 8$ & & \\
\hline \multirow[t]{3}{*}{$\operatorname{sed} n$} &.$£ 8$ & .18 & 085al6 & $s$ \\
\hline & & .06 & & \\
\hline & & .58 & & \\
\hline (Itan dssy 미 cosng & & .25 & & \\
\hline 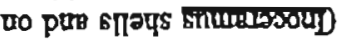 & & .09 & & \\
\hline 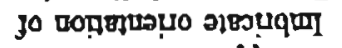 & & .16 & & \\
\hline ramoT pure sodd $\Omega$ &.$S L$ &.$Z L$ & 68IEZ6 & $\checkmark$ \\
\hline (1т़ा sny प्रा & & .96 & & \\
\hline s78ts คุก(ู) & & .011 & & \\
\hline radd $\Omega$ & .66 & .06 & E8IEl6 & $\varepsilon$ \\
\hline & & .291 & & \\
\hline (1rum jsSY 미 & & $.6 b 1$ & & \\
\hline 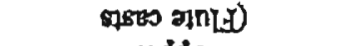 & & .891 & & \\
\hline $\operatorname{sid} n$ & .291 & $.0 L I$ & LZIEL6 & $\tau$ \\
\hline 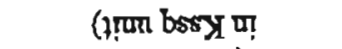 & & $.05\}$ & & \\
\hline spaq ssoso) & & .021 & & \\
\hline ramor & oEEI & .821 & 00IIGZ6 & I \\
\hline $\begin{array}{l}\text { Coloपा! purs } \\
\text { काmisar mold }\end{array}$ & 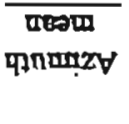 & 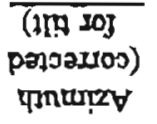 & $\begin{array}{c}\text {.00 } \\
\text { prग! }\end{array}$ & $\begin{array}{l}\overline{.00} \\
\text { dEyN }\end{array}$ \\
\hline 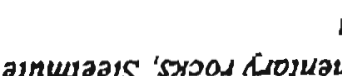 & & nO 8-0 p & o $\angle-a ' \angle$ & \\
\hline
\end{tabular}


Table 3. Rare Earth Element (REE) deferminations from selected igneous rocks in Sleetmute C-7, D-7, C-8, and D-8 Quadrangles, Alaska (all analyses in ppm)

\begin{tabular}{|c|c|c|c|c|c|c|c|c|c|c|}
\hline Map no. & 4 & 5 & 11 & $11 b$ & 15 & 17 & 19 & 21 & 26 & 34 \\
\hline Field no. & $90 G L 31$ & $90 \mathrm{BT} 40$ & $90 \mathrm{BT} 49$ & 90BT61 & 90BT81 & $80 \mathrm{BT} 83$ & $90 \mathrm{HA} 27$ & $90 \mathrm{BT} 121$ & $90 \mathrm{BT} 93$ & $90 \mathrm{BT} 115$ \\
\hline \multirow{2}{*}{ Rock type } & Basaltic & Vitric & Vitric & Basaltic & & Andesite & Vitric & Andesite & & \\
\hline & $\begin{array}{l}\text { Andesile } \\
\text { (TKvm) }\end{array}$ & $\begin{array}{c}\text { Porphyry } \\
\text { (Tkgp) }\end{array}$ & $\begin{array}{l}\text { Tuff } \\
\text { (TKat) }\end{array}$ & $\begin{array}{l}\text { Andesite } \\
\text { (TKro) }\end{array}$ & $\begin{array}{c}\text { Granodiorite } \\
\text { (TKsy) }\end{array}$ & $\begin{array}{l}\text { Tuff } \\
\text { (TKvi) }\end{array}$ & $\begin{array}{l}\text { Tuff } \\
\text { (TKai) }\end{array}$ & $\begin{array}{l}\text { Flow } \\
\text { (TKa) }\end{array}$ & $\begin{array}{c}\text { Granodiorite } \\
\text { (TKsy) }\end{array}$ & $\begin{array}{l}\text { Andesite } \\
\text { (TK₹a) }\end{array}$ \\
\hline $\mathrm{Ce}$ & 40.2 & 44.0 & 86.0 & 62.0 & 820 & 56.0 & 36.0 & 30.0 & 94.0 & 80.0 \\
\hline Eut & 1.0 & 10 & 2.0 & 1.0 & 15 & 2.0 & 0.5 & $\mathrm{ND}$ & 20 & 2.0 \\
\hline La & 14.0 & 18.0 & 39.0 & 24.0 & 35.0 & 230 & 15.0 & 9.0 & 40.0 & 32.0 \\
\hline Lu & 03 & $N D$ & 0.1 & 0.4 & 0.2 & 0.4 & $\mathrm{ND}$ & 0.1 & 0.6 & 0.1 \\
\hline Nd & 10.0 & 15.0 & 30.0 & 20.0 & 30.0 & 25.0 & 10.0 & 10.0 & 30.0 & 30.0 \\
\hline$S m$ & 4.4 & 45 & 65 & 5.8 & 6.4 & 53 & 2.3 & 24 & 6.3 & 6.5 \\
\hline $\mathrm{Tb}$ & 0.9 & $N D$ & 0.2 & 0.7 & 0.8 & 2.3 & $\mathrm{ND}$ & $\mathrm{ND}$ & 15 & 20 \\
\hline Th & 2.0 & 8.0 & 10.0 & 6.0 & 11.0 & 8.0 & 4.0 & 4.0 & 120 & 10.0 \\
\hline$U$ & 1.0 & 3.0 & 2.0 & 3.0 & 3.0 & 3.0 & 20 & ND & 20 & 4.0 \\
\hline $\mathrm{Yb}$ & 2.1 & $\mathrm{ND}$ & 0.8 & 2.6 & 1.3 & 3.0 & 0.1 & 0.8 & 1.6 & 2.9 \\
\hline
\end{tabular}

${ }^{1}$ Analybes by W.G. Armanin, Chemex Labs Ltd, Sparks, Nevada, using induced auclear activation techniques. 
Table 4. ${ }^{\circ} \mathrm{Ar}-{ }^{90} \mathrm{Ar}$ age determinations from selected igneous rocks in the Sleetmute C-7, C-8, D-7, and D-8 Quadrangles, Alaska'

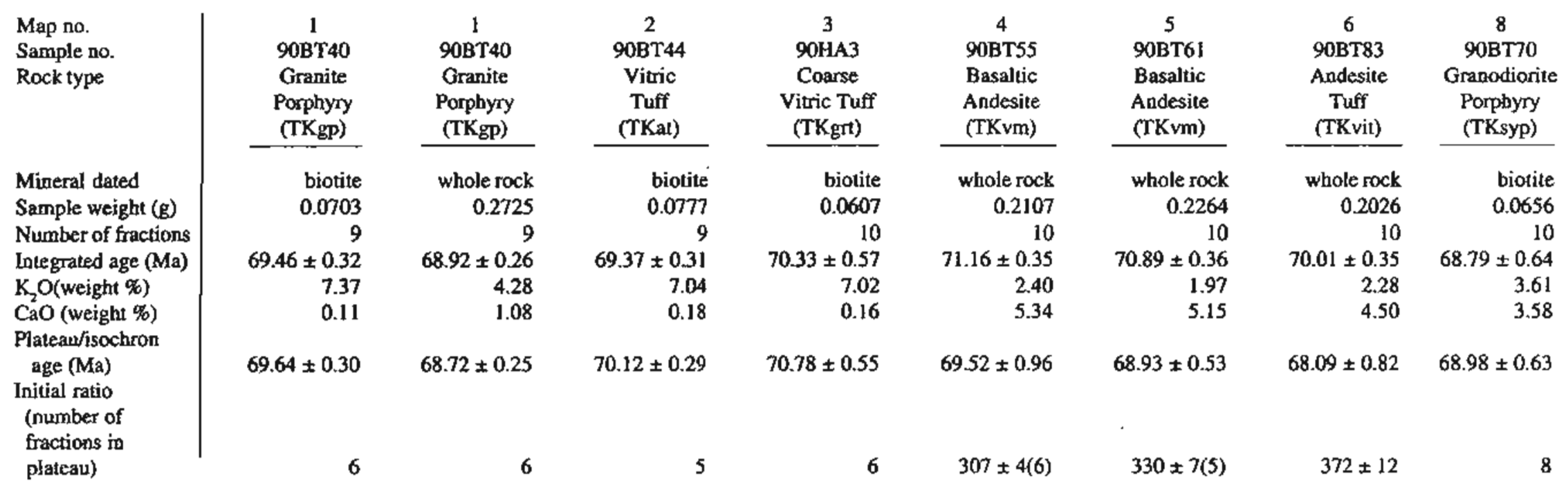

'Analyses by DT, Paul layer, DGGS-UAF Cooperative Geochronology Laboratory, Faibanks, Alaska. 
Table 5. $K$-As determination from granite porphyry pluton, Sleetmule C-7 Quadrangle, Alaskal

Map no.

Sample no.

Rock type

Mineral dated

Sample weight ( $(8)$

$\mathrm{K}_{2} \mathrm{O}$ (weight \%)

$40 \mathrm{AR}\left(10^{-11} \mathrm{~mol} / \mathrm{g}\right)$

${ }^{40} \mathrm{Ar}(\%)$

${ }^{40} \mathrm{Ar} / 40 \mathrm{~K} \times 10^{-3}$

Age (ma) ${ }^{2}$
13

87MDT25

granite porphyry (TKBp)

biotite

0.1144

8.822

93.2541

49.73

4.2667

$71.98 \pm 2.16$

${ }^{1}$ Analyses by Robin Cotrell and Donald L. Tumer, DGGS-

UAF Cooperative Geochmonology Laboratory, Fairbanks, Alaska.

${ }^{2}$ Contants used in age calculations

$$
\begin{aligned}
& \lambda_{\mathrm{e}}=0.581 \times 10^{-10} \mathrm{yr}^{1} \\
& \lambda_{\mathrm{B}}=4.962 \times 10^{-10} \mathrm{yr}^{1} \\
& 40 \mathrm{~K} / \mathrm{K}_{\text {total }}=1.167 \times 10^{-4} \mathrm{~mol} / \mathrm{mol}
\end{aligned}
$$




\begin{tabular}{|c|c|c|c|c|c|}
\hline $\begin{array}{l}\text { Map } \\
\text { no. }\end{array}$ & $\begin{array}{c}\text { Field } \\
\text { no. }\end{array}$ & $\begin{array}{c}\text { Laboratory } \\
\text { no. }\end{array}$ & $\begin{array}{c}\text { C14 age } \\
\text { (years B. } \pm 15 \text { ) }\end{array}$ & $\mathrm{C} 13 / \mathrm{C}_{12}$ & $\begin{array}{c}\mathrm{Cl3} \\
\text { adjusted age }\end{array}$ \\
\hline $7 a$ & $90 \mathrm{BT} 71$ & Beta 40059 & $1900 \pm 41 \mathrm{BP}$ & NA & NA \\
\hline $7 b$ & 90BT75 & Beta 40060 & $13,600 \pm 293$ BP. & NA & NA \\
\hline $\begin{array}{l}10 \\
11\end{array}$ & $\begin{array}{l}91 \mathrm{BT1} \\
92 \mathrm{BT} 4 \mathrm{a} \\
92 \mathrm{BT} 4 \mathrm{~b} \\
91 \mathrm{BT} 4 \mathrm{c}\end{array}$ & $\begin{array}{l}\text { Beta } 47634 \\
\text { Beta } 47635 \\
\text { Beta } 47636 \\
\text { Beta } 47367\end{array}$ & $\begin{array}{l}23,690 \pm 510 \text { B.P. } \\
33,800 \pm 2,000 \text { B.P. } \\
34,710 \pm 1,240 \text { B.P. } \\
\geq 39,600 \mathrm{~B}, \mathrm{P} .\end{array}$ & $\begin{array}{l}-26.9 \\
-26.6 \\
-28.5 \\
-27.2\end{array}$ & $\begin{array}{l}23,660 \pm 510 \text { B.P. } \\
33,770 \pm 2,000 \text { B.P. } \\
34,650 \pm 1,240 \text { B.P. } \\
\text { NA }\end{array}$ \\
\hline $\begin{array}{l}12 \\
14\end{array}$ & $\begin{array}{l}91 \mathrm{BT5b} \\
91 \mathrm{BT31a} \\
91 \mathrm{BT} 3 \mathrm{tb}\end{array}$ & $\begin{array}{l}\text { Beta } 47638 \\
\text { Beta } 47639 \\
\text { Beta } 47640\end{array}$ & $\begin{array}{l}\geq 38,600 \text { B.P. } \\
11,760 \pm 70 \text { B.P. } \\
16,410 \pm 150 \text { BP }\end{array}$ & $\begin{array}{l}-27.2 \\
-26.8 \\
-26.9\end{array}$ & $\begin{array}{l}\text { NA } \\
11,730 \pm 70 \text { B.P. } \\
16,380 \pm 150 \text { B.P. }\end{array}$ \\
\hline 15 & $\begin{array}{l}91 \mathrm{BT} 32 \mathrm{a} \\
91 \mathrm{BT} 32 \mathrm{~b}\end{array}$ & $\begin{array}{l}\text { Beta } 47641 \\
\text { Beta } 47642\end{array}$ & $\begin{array}{l}\geq 40,920 \text { B.P. } \\
26,446 \pm 530 \text { B.P. }\end{array}$ & $\begin{array}{l}-28.3 \\
-27.6\end{array}$ & $\begin{array}{l}\text { NA } \\
26,400 \pm 530 \text { BP. }\end{array}$ \\
\hline 16 & 91BT86 & Beta 47643 & $16,670 \pm 360$ B.P. & -272 & $16,640 \pm 360$ B.P. \\
\hline
\end{tabular}

Palsa on till of Tolstoi Lake age on upper Getmuna Creek at $2,150 \mathrm{ft}$ elevation; sample taken from $1.5 \mathrm{~m}$ depth on top of blue, varved, lacustrine deposit.

From organic peat layer in till of Tolstoi Lake age in upper Getmuna Creek at $2,150 \mathrm{ft}$ elevation; sample taken $2 \mathrm{~m}$ below $90 \mathrm{BT} 7 \mathrm{l}$ (see figme $\mathrm{x}$ ).

From organic loess $6 \mathrm{~m}$ below surface on top Jungiuk Creek outwash far. 9lBT4a from peat layer $3 \mathrm{M}$ below surface

9 IBT4b from top of thin outwash $1 \mathrm{~m}$ below $91 \mathrm{BT} 4 \mathrm{a}$

91BT4c from peat layer beiow ontwash

All samples from along west bank Kuskokwim River in NE comer of Sleetmute C-7 Quadrangle.

From bluish clay layer in outwash gravel $1 / 2 \mathrm{~km}$ below 91BT4a-c site.

91BT31a from forest hayer $12 \mathrm{~m}$ below loess cap

91BT3 ib from peat layer is m below 91BT31a, about $5 \mathrm{~km}$ below mouth of Sue Creek, Kuskokwim River.

91BT32a from organic silt horizon $0.3 \mathrm{~m}$ above outwash gravel $6 \mathrm{~m}$ below modern forest layer

91BT32b from organic pent $3 \mathrm{~m}$ below modern forest layer, and about $3 \mathrm{~m}$ above 91BT32a; site is $1 \mathrm{~km}$ below 91BT31 sample sites.

From organic sill layer directly undemeath sandy eolian dune complex; about $6 \mathrm{~km}$ dowariver fron Napamiut.

'Analytical work by Beta Analytic Inc. Coral Gables Flordia, 33124; dates are reported as RCYBP (radiocabon years before 1950 AD). Half-life of radiocarbon is 5,568 years; accuracy of results are one standard deviation. Adjusted ages are nomalized to -25 per mil carbon 13 . 
Table 2. Major oxide and trace element determinations and CIPW normative mineralogy for selected igneous rocks from Sleetmute C-7, C-8, D-7, and D.8 Quadrangles - page I of 5

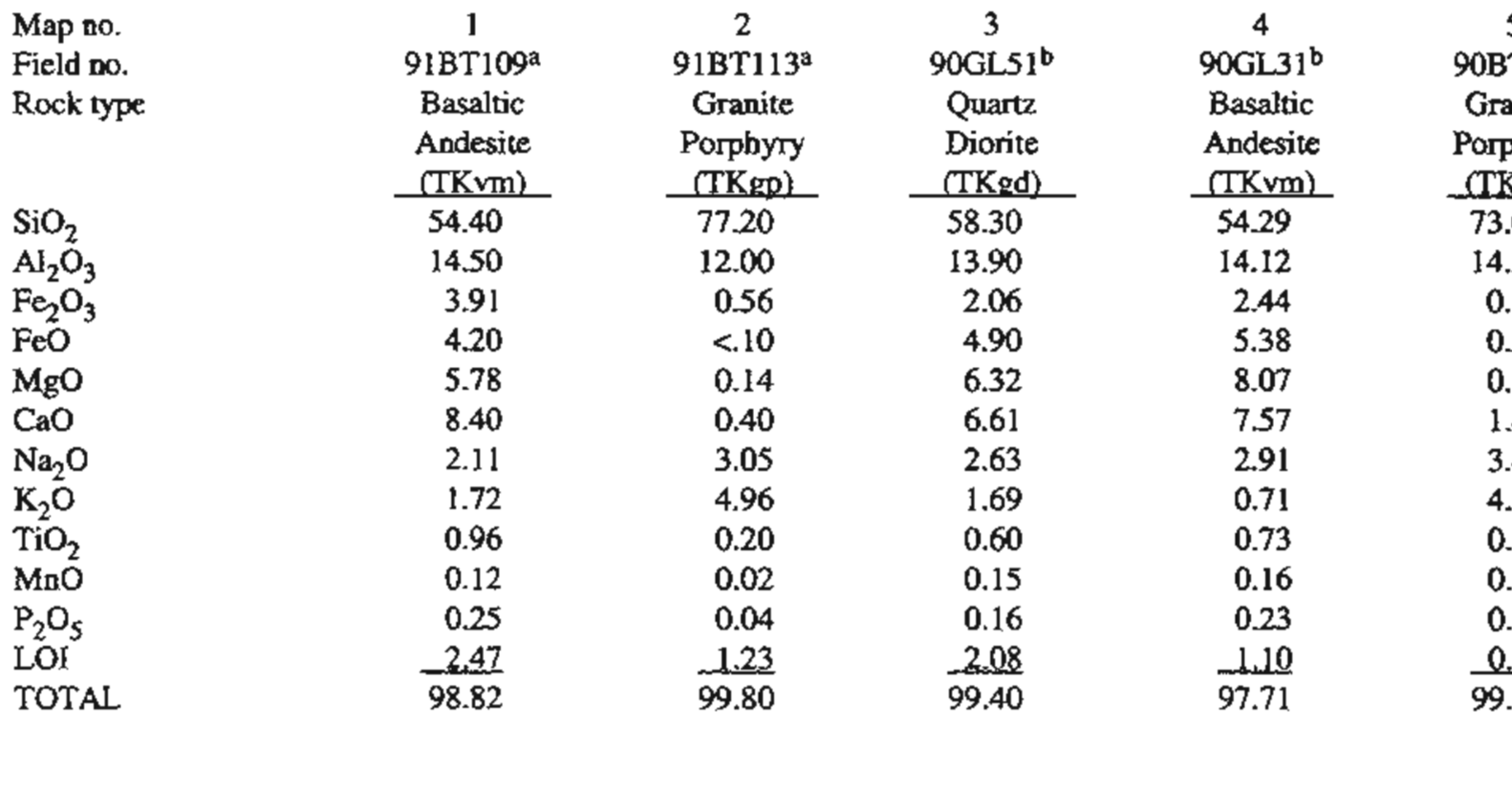

5
GTanite
Orphyry
TKop)
73.07
14.30
0.82
0.51
0.17
1.44
3.42
4.60
0.07
0.01
0.12
0.65
99.18

\begin{tabular}{|c|c|}
\hline 6 & 7 \\
\hline $90 \mathrm{HA}^{6}{ }^{b}$ & $90 \mathrm{~B}^{1} 41 \mathrm{~A}^{\mathrm{b}}$ \\
\hline Coarse & Coarse \\
\hline Vitric Tuff & Vitric Tuff \\
\hline (TK art) & (TKat) \\
\hline 75.00 & 59.83 \\
\hline 12.70 & 17.48 \\
\hline 0.93 & 2.64 \\
\hline 1.10 & 3.37 \\
\hline 0.66 & 3.08 \\
\hline 1.51 & 4.97 \\
\hline 230 & 3.88 \\
\hline 3.22 & 3.27 \\
\hline 0.40 & 0.88 \\
\hline 0.03 & 0.05 \\
\hline 0.10 & 0.38 \\
\hline 1.77 & 0.24 \\
\hline 99.72 & 1 \\
\hline
\end{tabular}

\begin{tabular}{|c|c|}
\hline 8 & 9 \\
\hline $90 \mathrm{HA} 4^{b}$ & $90 \mathrm{HA} 6^{6}$ \\
\hline Basaltic & Vesicular \\
\hline Andesite & Andesite \\
\hline (TKvm) & STKgyml \\
\hline 56.60 & 59.20 \\
\hline 15.10 & 16.00 \\
\hline 2.28 & 2.01 \\
\hline 5.10 & 5.00 \\
\hline 5.98 & 3.03 \\
\hline 6.53 & 5.36 \\
\hline 2.72 & 3.04 \\
\hline 1.79 & 2.76 \\
\hline 1.14 & 1.37 \\
\hline 0.13 & 0.12 \\
\hline 0.24 & 0.33 \\
\hline 2.70 & 1.77 \\
\hline 0.31 & 99.99 \\
\hline
\end{tabular}

\begin{tabular}{c}
10 \\
90HA $7^{\mathrm{b}}$ \\
Latite \\
Tuff \\
(TKdd) \\
\hline 68.00 \\
13.60 \\
1.75 \\
2.30 \\
0.83 \\
2.47 \\
3.38 \\
3.83 \\
0.73 \\
0.06 \\
0.16 \\
2.40 \\
\hline 99.51
\end{tabular}

$\stackrel{11}{90 \mathrm{BT} 49^{\mathrm{b}}}$

12

$\begin{array}{lrrr}\mathrm{Cr} & 249 & 410 & 507 \\ \mathrm{Rb} & 62 & 158 & 51 \\ \mathrm{Sr} & 533 & 40 & 418 \\ \mathrm{Y} & 19 & 23 & 15 \\ \mathrm{Zr} & 129 & 171 & 142 \\ \mathrm{Nb} & 21 & 21 & 20 \\ \mathrm{Ba} & 1.530 & 1,820 & 1,620\end{array}$

$\begin{array}{ll}\text { NA } & \text { NA } \\ \text { NA } & \text { NA } \\ \text { NA } & \text { NA } \\ \text { NA } & \text { NA } \\ \text { NA } & \text { NA } \\ \text { NA } & \text { NA } \\ \text { NA } & \text { NA }\end{array}$

$\begin{array}{rrr}41 & \text { NA } & 384 \\ 94 & \text { NA } & 86 \\ 237 & \text { NA } & 359 \\ <10 & \text { NA } & 17 \\ 144 & \text { NA } & 222 \\ 19 & \text { NA } & 12 \\ 1,900 & \text { NA } & 799\end{array}$

$\begin{array}{rr}384 & 101 \\ 86 & 94 \\ 359 & 332 \\ 17 & 33 \\ 222 & 286 \\ 12 & 18 \\ 799 & 1,060\end{array}$

29
147
178
46
366
21
1490

$\begin{array}{lr}\text { NA } & 343 \\ \text { NA } & 65 \\ \text { NA } & 309 \\ \text { NA } & 33 \\ \text { NA } & 291 \\ \text { NA } & <10 \\ \text { NA } & 900\end{array}$

Quarz
Corundurs
Orthoclase
Albite
Anorthite
Diopside
Olivine
Hyperstene
Magnedite
Imenite
Aparite
TOTAL

$\begin{array}{r}11.77 \\ 0.00 \\ 10.60 \\ 18.62 \\ 26.09 \\ 12.48 \\ 0.00 \\ 12.73 \\ 5.22 \\ 1.90 \\ -0.60 \\ \hline 100.01\end{array}$

$\begin{array}{rr}40.10 & 12.91 \\ 1.00 & 0.00 \\ 29.73 & 10.32 \\ 26.18 & 22.99 \\ 1.75 & 21.83 \\ 0.00 & 8.85 \\ 0.00 & 0.00 \\ 0.35 & 19.25 \\ 0.57 & 2.28 \\ 0.22 & 1.18 \\ 0.09 & \underline{0.38} \\ 99.99 & 99.99\end{array}$

$\begin{array}{lr}\text { NA } & 32.98 \\ \text { NA } & 1.39 \\ \text { NA } & 27.60 \\ \text { NA } & 29.39 \\ \text { NA } & 6.46 \\ \text { NA } & 0.00 \\ \text { NA } & 0.00 \\ \text { NA } & 0.65 \\ \text { NA } & 1.12 \\ \text { NA } & 0.14 \\ \text { NA } & \underline{0.28} \\ \text { NA } & 100.01\end{array}$

CIPW Norms (weightpercent)

Differentauon

Index

40.99

96.01

46.22

NA

\begin{tabular}{rr}
46.02 & 9.63 \\
2.99 & 0.00 \\
19.45 & 19.43 \\
19.89 & 33.01 \\
6.99 & 20.73 \\
0.00 & 1.26 \\
0.00 & 0.00 \\
2.44 & 10.08 \\
1.20 & 3.31 \\
0.78 & 1.68 \\
0.24 & -0.89 \\
\hline 100.00 & 100.02
\end{tabular}

10.83
0.00
10.91
23.74
24.45
5.92
0.00
18.77
2.57
2.23
0.57
99.99

$\begin{array}{r}14.07 \\ 0.00 \\ 16.70 \\ 26.34 \\ 22.38 \\ 2.17 \\ 0.00 \\ 12.74 \\ 2.17 \\ 2.66 \\ 0.78 \\ \hline 100.01\end{array}$

27.66
0.00
23.35
29.50
10.96
0.50
0.00
3.97
2.24
1.43
0.38
99.99

$\begin{array}{rr}41.49 & 10.24 \\ 3.52 & 0.00 \\ 16.06 & 13.34 \\ 21.07 & 24.51 \\ 11.36 & 23.36 \\ 0.00 & 6.00 \\ 0.00 & 0.00 \\ 2.35 & 15.68 \\ 2.57 & 4.27 \\ 1.03 & 2.10 \\ 0.55 & 0.50 \\ 100.00 & 100.00\end{array}$

Plagioclase

58.35

6.26

NA

26.00

38.58

50.74

45.94

80.51

78.62

48.09

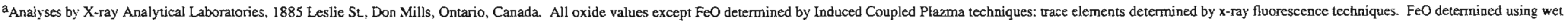
cherristy rechnique.
bAnalyses by W.G. Amanin, Chemex Labs Inc., Sparks, Nevadza all oxide samples determined by Induced Coupled Piazma techniques; trace ejements by $x$-ray fluorescence techniques; FeO by wet chemistry. 
Table 2. Major oxide and trace element determinations and CIPW nommative mineralogy for selected igneous rocks from Sleetmute C-7, C-8, D-7, and D-8 Quadrangles - Continued - page 2 of 5

\begin{tabular}{|c|c|c|c|c|c|c|c|c|c|c|c|c|}
\hline \multirow{4}{*}{$\begin{array}{l}\text { Map no. } \\
\text { Field no. } \\
\text { Rock type }\end{array}$} & 13 & 14 & 15 & 16 & 17 & 18 & 19 & 20 & 21 & 22 & 23 & 24 \\
\hline & $90 \mathrm{BT} 61^{\mathrm{b}}$ & $90 \mathrm{~B} T 63^{2}$ & $91 \mathrm{BT73^{ \textrm {a } }}$ & 91BT119a & $92 \mathrm{BTL} 20^{\mathrm{A}}$ & $90 \mathrm{BT}^{\mathrm{T}} 3 \mathrm{~B}^{\mathrm{a}}$ & $90 \mathrm{BT} 81^{\mathrm{b}}$ & $91 \mathrm{BT} 101^{\mathrm{a}}$ & $90 \mathrm{BT}^{2} 3^{\mathrm{b}}$ & $90 \mathrm{BT} 70^{\mathrm{b}}$ & $90 \mathrm{HA} 27^{\mathrm{b}}$ & $90 \mathrm{BT} T 85 \mathrm{~B}$ \\
\hline & Basaltic & Basaltic & High K & Basaltic & Vitreous & Andesite & Granodiorite & Basaltic & Andesite & Granodiorite & Vitric & Andesite \\
\hline & Andesite & Andesite & Andesite & Andesite & Andesite flow & Tuff & (TKsy) & Andesite & Flow & Porphyry & Lithic Tuff & (TKdt) \\
\hline & (TKprm) & (TKrn) & $(\mathrm{TKdt})$ & (TKvm) & (TKvv) & (TKd) & & _(IKrm) & TKaL & (TKSYD $)$ & (TKat) & \\
\hline $\mathrm{SiO}_{2}$ & 56.27 & 57.70 & 62.20 & 58.40 & 61.40 & 62.00 & 65.45 & 56.00 & 56.09 & 65.77 & 73.71 & 62.19 \\
\hline $\mathrm{Al}_{2} \mathrm{O}_{3}$ & 15.62 & 15.40 & 14.70 & 16.30 & 15.80 & 15.80 & 14.77 & 15.30 & 14.72 & 15.14 & 13.92 & 16.56 \\
\hline $\mathrm{Fe}_{2} \mathrm{O}_{3}$ & 2.47 & 2.55 & 2.34 & 273 & 6.05 & 209 & 0.82 & 2.96 & 2.80 & 1.60 & 0.79 & 2.39 \\
\hline $\mathrm{FeO}$ & 4.46 & 4.20 & 4.40 & 2.90 & 1.50 & 3.60 & 3.28 & 4.30 & 4.02 & 2.90 & 0.16 & 3.67 \\
\hline $\mathrm{MgO}$ & 5.66 & 5.11 & 1.12 & 3.84 & 1.85 & 1.46 & 2.10 & 4.80 & 4.56 & 2.44 & 0.51 & 1.62 \\
\hline $\mathrm{CaO}$ & 6.26 & 5.83 & 3.65 & 6.84 & 4.04 & 4.04 & 2.88 & 6.44 & 5.89 & 3.32 & 1.14 & 4.17 \\
\hline $\mathrm{Na}_{2} \mathrm{O}$ & 3.11 & 2.52 & 3.94 & 2.94 & 3.68 & 4.45 & 2.79 & 2.59 & 2.83 & 3.14 & 3.63 & 4.11 \\
\hline $\mathrm{K}_{2} \mathrm{O}$ & 1.99 & 2.21 & 3.18 & 204 & 3.05 & 2.11 & 3.29 & 1.90 & 2.31 & 3.45 & 2.82 & 3.00 \\
\hline $\mathrm{TiO}_{2}$ & 1.05 & 1.06 & 1.21 & 0.75 & 1.03 & 0.94 & 0.66 & 1.31 & 1.10 & 0.78 & 0.11 & 0.99 \\
\hline $\mathrm{MnO}$ & 0.10 & 0.12 & 0.11 & 0.09 & 0.09 & 0.11 & 0.06 & 0.12 & 0.13 & 0.04 & 0.01 & 0.05 \\
\hline $\mathrm{P}_{2} \mathrm{O}_{5}$ & 0.28 & 0.22 & 0.34 & 0.23 & 0.24 & 0.24 & 0.28 & 0.28 & 0.35 & 0.28 & 0.13 & 0.32 \\
\hline LOI & +44 & 1.93 & -2.85 & 2.08 & 1.20 & 2.54 & $\underline{2.70}$ & 3.23 & 4.14 & 1.83 & 2.10 & 1.26 \\
\hline TOTAL & 98.71 & 98.85 & 100.04 & 99.14 & 100.63 & 99.38 & 99.08 & 99.23 & 98.94 & 100.69 & 99.03 & 100.33 \\
\hline
\end{tabular}

$\begin{array}{lrrrr}\mathrm{Cr} & \text { NA } & 349 & <10 & 17 \\ \mathrm{Rb} & \mathrm{NA} & 85 & 178 & 6 \\ \mathrm{Sr} & \text { NA } & 348 & 232 & 57 \\ \mathrm{Y} & \text { NA } & 27 & 68 & 10 \\ \mathrm{Zr} & \text { NA } & 266 & 380 & 136 \\ \mathrm{Nb} & \text { NA } & 18 & 20 & 25 \\ \text { Ba } & \text { NA } & 916 & 1,450 & 1,79\end{array}$

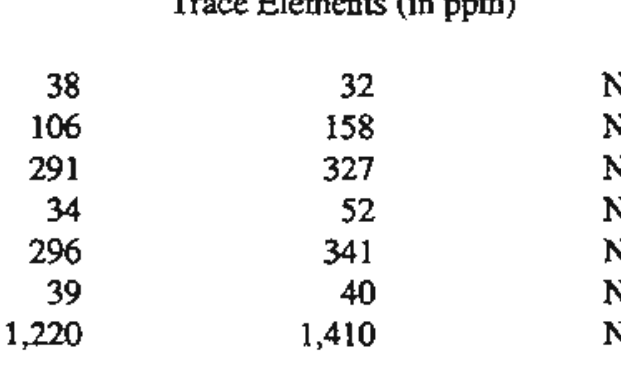

Quartz
Conndum

Cormaclase

Albite

Anoritite

Diopside

Olivine

Hyperstone

Magnetite

Apatite

Differentation

Index

\begin{tabular}{cc}
8.91 & 14.29 \\
0.00 & 0.00 \\
12.15 & 13.54 \\
27.09 & 22.11 \\
23.54 & 25.07 \\
5.34 & 2.75 \\
0.00 & 0.00 \\
17.17 & 16.50 \\
2.97 & 3.14 \\
2.06 & 2.09 \\
0.67 & 0.53 \\
\hline 100.10 & 100.02
\end{tabular}

17.62

0.00
19.43

34.47

13.48

2.49
0.00

6.54
2.79

2.38

$\frac{0.81}{100.01}$

$\begin{array}{lr}\text { NA } & 194 \\ \text { NA } & 44 \\ \text { NA } & 355 \\ \text { NA } & 38 \\ \text { NA } & 262 \\ \text { NA } & 31 \\ \text { NA } & 882\end{array}$

NA
NA
NA
NA
NA
NA
NA

NA
NA
NA
NA
NA
NA
NA

$\begin{array}{ll}\text { NA } & \text { NA } \\ \text { NA } & \text { NA } \\ \text { NA } & \text { NA } \\ \text { NA } & \text { NA } \\ \text { NA } & \text { NA } \\ \text { NA } & \text { NA } \\ \text { NA } & \text { NA }\end{array}$

CIPW Norms (weight/percent)

Clagioclase

49.94

71.52

14.76
0.00
12.46
25.71
26.11
5.90
0.00
9.42
3.61
1.47
0.55
99.99

18.32

0.00
18.57
32.08

32.08
18.12

18.12
0.71
0.00

0.00
4.42

4.42
5.21
2.02

2.02
0.52
100.02

\begin{tabular}{cc}
17.07 & 27.16 \\
0.00 & 2.12 \\
12.93 & 20.03 \\
39.04 & 24.32 \\
17.53 & 12.84 \\
1.35 & 0.00 \\
0.00 & 0.00 \\
7.11 & 9.35 \\
2.54 & 2.35 \\
1.85 & 1.17 \\
0.58 & 0.66 \\
\hline 100.00 & 100.00
\end{tabular}

$27.16 \quad 13.54$

$1354 \quad 12.89$

$12.89 \quad 23.29$

$\begin{array}{lll}23.29 & 40.21 & 15.10\end{array}$

$\begin{array}{lll}11.75 & 14.34 & 20.69 \\ 22.94 & 2516 & 2696\end{array}$

20.69
26.96

25.66
4.70

25.16

21.68
5.28

5.28
0.00

0.00
14.34

$\begin{array}{r}3.78 \\ 2.60 \\ 0.68 \\ \hline 99.99\end{array}$

$\frac{0.52}{100.02}$

4.84

\begin{tabular}{c}
2.20 \\
0.85 \\
\hline
\end{tabular}

14.86
0.00

0.00

0.00
9.27
1.88
1.50

$\frac{0.66}{100.00}$

\begin{tabular}{cc}
40.21 & 15.10 \\
3.23 & 0.00 \\
17.19 & 17.97 \\
31.69 & 35.24 \\
4.96 & 18.12 \\
0.00 & 0.60 \\
0.00 & 0.00 \\
1.31 & 7.39 \\
0.97 & 2.92 \\
0.22 & 1.91 \\
0.31 & 0.75 \\
\hline 100.00 & 100.00
\end{tabular}

68.97

69.04

71.52

48.23

52.39

70.94

89.10

68.31

46.40

28.11

50.39

36.10

30.99

34.55

52.80

46.29

35.53

13.53

33.95 
Table 2. Major oxide and trace element determinations and CIPW normarive mineralogy for selected igneous rocks from Sleetmute C-7, C-8, D-7, and D-8 Quadrangles - Continued - page 3 of 5

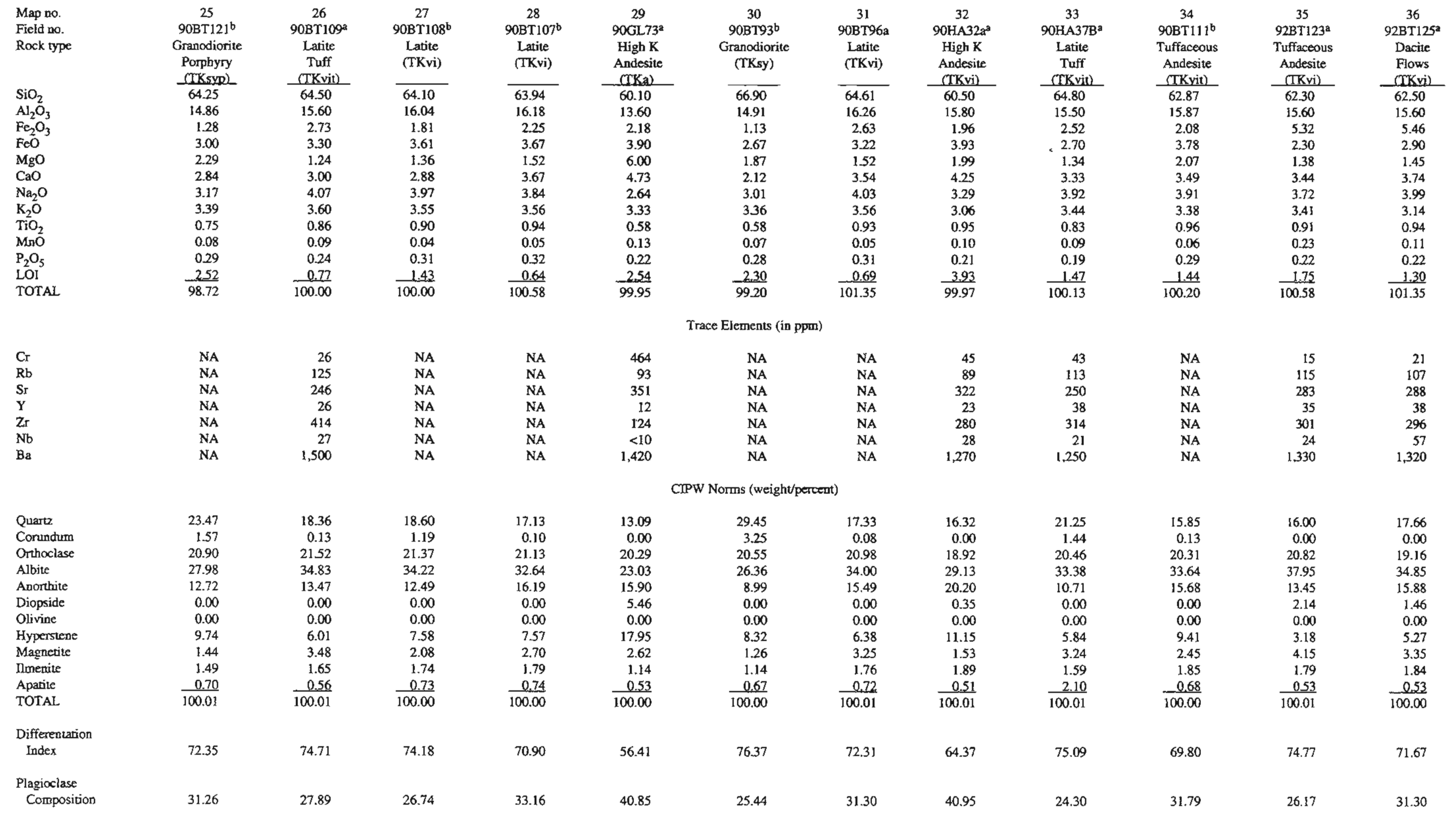


Table 2. Major oxide and trace element determinations and CIPW normative mineralogy for selected igneous rocks from Sleetmute C-7, C-8, D-7, and D-8 Quadrangles * Continued - page 4 of 5

\begin{tabular}{|c|c|c|c|c|c|c|c|c|c|c|c|c|}
\hline $\begin{array}{l}\text { Map no. } \\
\text { Field no }\end{array}$ & $\begin{array}{c}37 \\
90 \mathrm{BTI} 14^{\mathrm{b}}\end{array}$ & $\begin{array}{c}38 \\
91 G L 27^{a}\end{array}$ & $\begin{array}{c}39 \\
91 G L 26^{3}\end{array}$ & $\begin{array}{c}40 \\
90 \mathrm{BT} 115^{\mathrm{b}}\end{array}$ & $\begin{array}{c}41 \\
92 \mathrm{BT} 10 \mathrm{I}^{\mathrm{a}}\end{array}$ & $\begin{array}{c}42 \\
91 \mathrm{BT} 42^{\mathrm{a}}\end{array}$ & $\begin{array}{c}43 \\
92 \mathrm{GL} 86^{\mathrm{a}}\end{array}$ & $\begin{array}{c}44 \\
91 \mathrm{BT} 35^{\mathrm{a}}\end{array}$ & $\begin{array}{c}45 \\
91 \mathrm{BT} 34 \mathrm{a}^{\mathrm{a}}\end{array}$ & $\begin{array}{c}46 \\
91 \mathrm{BT} 34 \mathrm{~b}^{\mathrm{a}}\end{array}$ & $\begin{array}{c}47 \\
91 \mathrm{BT} 34 \mathrm{c}^{\mathrm{a}}\end{array}$ & $\begin{array}{c}48 \\
91 \mathrm{BT} 16^{\mathrm{a}}\end{array}$ \\
\hline Rock type & $\begin{array}{c}\text { Latile } \\
\text { Tuff } \\
\text { (TKfit) } \\
\end{array}$ & $\begin{array}{c}\text { Latite } \\
\text { Tuff } \\
\text { (TKfi) }\end{array}$ & $\begin{array}{l}\text { Andesite } \\
\text { (TKvi) }\end{array}$ & $\begin{array}{l}\text { Andessite } \\
\text { (TKva) }\end{array}$ & $\begin{array}{c}\text { Andesite } \\
\text { (Kac) }\end{array}$ & $\begin{array}{l}\text { Granodiorite } \\
\text { (TKsyp) }\end{array}$ & $\begin{array}{l}\text { Andesite } \\
\text { (Kac) }\end{array}$ & $\begin{array}{c}\text { Mafic } \\
\text { flow } \\
\text { (TKVm) }\end{array}$ & $\begin{array}{c}\text { Mafic } \\
\text { flow } \\
\text { (TKvm) }\end{array}$ & $\begin{array}{c}\text { Mafic } \\
\text { flow } \\
\text { (TKvm) }\end{array}$ & $\begin{array}{l}\text { Latite } \\
\text { Tuff } \\
(T K d t) \\
\end{array}$ & $\begin{array}{l}\text { Gabbro } \\
\text { (TKgb) }\end{array}$ \\
\hline $\mathrm{SiO}_{2}$ & $\overline{64.54}$ & 64.30 & 62.80 & 61.85 & 57.60 & 67.70 & 55.10 & 54.70 & 54.00 & 57.20 & 63.00 & 53.00 \\
\hline $\mathrm{Al}_{2} \mathrm{O}_{3}$ & 16.31 & 15.90 & 15.90 & 15.95 & 17.70 & 14.70 & 18.70 & 14.70 & 14.40 & 15.60 & 15.40 & 14.70 \\
\hline $\mathrm{Fe}_{2} \mathrm{O}_{3}$ & 3.25 & 1.87 & 2.85 & 2.46 & 6.22 & 1.12 & 6.65 & 2.43 & 2.74 & 3.44 & 2.14 & 2.63 \\
\hline $\mathrm{FeO}$ & 2.46 & 3.80 & 3.00 & 3.48 & 2.30 & 2.70 & 2.00 & 5.40 & 4.80 & 4.00 & 3.20 & 4.60 \\
\hline $\mathrm{MgO}$ & 1.38 & 1.40 & 1.53 & 1.73 & 2.32 & 1.19 & 2.50 & 8.22 & 8.34 & 5.27 & 1.40 & 6.96 \\
\hline $\mathrm{CaO}$ & 3.44 & 3.41 & 3.89 & 3.87 & 5.25 & 2.41 & 3.74 & 6.85 & 6.91 & 5.95 & 3.42 & 7.10 \\
\hline $\mathrm{Na}_{2} \mathrm{O}$ & 3.97 & 3.85 & 3.80 & 3.84 & 3.77 & 3.69 & 4.34 & 2.41 & 2.34 & 3.10 & 3.77 & 2.46 \\
\hline $\mathrm{K}_{2} \mathrm{O}$ & 3.69 & 3.54 & 3.22 & 3.31 & 2.20 & 4.39 & 2.44 & 1.16 & 1.09 & 2.05 & 3.02 & 1.30 \\
\hline $\mathrm{TiO}_{2}$ & 0.95 & 0.91 & 0.96 & 0.93 & 0.86 & 0.74 & 1.18 & 0.70 & 0.66 & 1.19 & 0.87 & 0.91 \\
\hline MnŌ & 0.05 & 0.10 & 0.11 & 0.09 & 0.14 & 0.08 & 0.23 & 0.14 & 0.13 & 0.12 & 0.09 & 0.15 \\
\hline $\mathrm{P}_{2} \mathrm{O}_{5}$ & 0.32 & 0.22 & 0.24 & 0.37 & 0.30 & 0.14 & 0.18 & 0.13 & 0.13 & 0.24 & 0.21 & 0.18 \\
\hline LOI & 0.74 & 0.77 & 1.39 & 0.90 & 1.45 & 0.85 & 3.65 & 3.23 & 3.31 & - & 3.47 & 5.23 \\
\hline TOTAL & 101.10 & 100.07 & 99.69 & 98.78 & 100.11 & 99.71 & 100.71 & 100.07 & 98.85 & 100.09 & 99.99 & 99.22 \\
\hline \multicolumn{13}{|c|}{ Trace Elements (in ppm) } \\
\hline $\mathrm{Cr}_{\mathrm{r}}$ & $\mathrm{NA}$ & 28 & 27 & NA & $<10$ & 38 & $<10$ & 686 & 643 & 281 & 39 & 480 \\
\hline $\mathbf{R b}$ & NA & 123 & 99 & NA & 61 & 156 & 83 & 34 & 33 & 72 & 144 & 54 \\
\hline$S_{T}$ & $\mathrm{NA}$ & 299 & 294 & NA & 552 & 167 & 469 & 318 & 361 & 338 & 272 & 459 \\
\hline $\mathrm{Y}$ & $\mathrm{NA}$ & 20 & 42 & $\mathrm{NA}$ & 15 & 45 & 26 & 16 & 22 & 40 & 38 & 18 \\
\hline $\mathrm{Zr}$ & $\mathrm{NA}$ & 374 & 383 & NA & 132 & 423 & 147 & 109 & 92 & 251 & 381 & 154 \\
\hline $\mathrm{Nb}$ & NA & 25 & 11 & $\mathrm{NA}$ & 19 & 29 & 29 & 16 & 13 & 14 & 31 & 22 \\
\hline $\mathbf{B a}$ & NA & 1,460 & 1,390 & NA & 1,740 & 1,580 & 2,190 & 599 & 482 & 891 & 1.448 & 788 \\
\hline \multicolumn{13}{|c|}{ CIPW Norms (weight/percent) } \\
\hline Quarz & 1858 & 17.76 & 18.07 & 15.98 & 13.91 & 21.85 & 9.79 & 8.13 & 8.72 & 11.39 & 20.04 & 8.61 \\
\hline Corundum & 0.30 & 0.06 & 0.00 & 0.00 & 0.30 & 0.00 & 2.69 & 0.00 & 0.00 & 0.00 & 0.22 & 0.00 \\
\hline Orthoclase & 21.73 & 21.16 & 19.42 & 20.06 & 13.53 & 26.32 & 15.20 & 7.12 & 6.76 & 12.40 & 1856 & 8.22 \\
\hline Albite & 33.47 & 32.94 & 32.82 & 33.33 & 33.19 & 31.68 & 38.72 & 21.19 & 20.79 & 26.84 & 33.17 & 22.27 \\
\hline Anorthite & 14.92 & 15.66 & 17.17 & 16.93 & 25.06 & 10.74 & 18.32 & 26.88 & 26.85 & 23.12 & 36.22 & 26.99 \\
\hline Diopside & 0.00 & 0.00 & 0.76 & 0.23 & 0.00 & 0.39 & 0.00 & 6.06 & 6.70 & 4.36 & 0.00 & 7.55 \\
\hline Olivine & 0.00 & 0.00 & 0.00 & 0.00 & 0.00 & 0.00 & 0.00 & 0.00 & 0.00 & 0.00 & 0.00 & 0.00 \\
\hline Hyperstene & 3.78 & 8.04 & 5.61 & 7.70 & 6.06 & 6.07 & 6.57 & 26.17 & 24.88 & 14.56 & 6.88 & 20.78 \\
\hline Magnetite & 4.70 & 2.13 & 3.73 & 3.09 & 5.54 & 1.21 & 5.91 & 2.77 & 3.37 & 4.45 & 2.70 & 3.29 \\
\hline Mmenite & 1.80 & 1.75 & 1.86 & 1.81 & 1.68 & 1.43 & 2.36 & 1.36 & 1.66 & 2.31 & 1.72 & 1.85 \\
\hline Aparite & 0.74 & 0.52 & 0.57 & 0.88 & 0.72 & 0.33 & 0.44 & 0.31 & 0.27 & 0.57 & 0.51 & 0.45 \\
\hline TOTAL & 100.00 & 100.02 & 100.01 & 100.01 & 99.99 & 100.02 & 100.00 & 99.99 & 100.00 & 100.00 & 100.02 & 100.01 \\
\hline \multicolumn{13}{|l|}{ Differentation } \\
\hline Index & 73.77 & 71.86 & 70.31 & 69.36 & 60.63 & 79.85 & 63.71 & 36.44 & 36.27 & 50.63 & 71.77 & 39.10 \\
\hline \multicolumn{13}{|l|}{ Plagioclase } \\
\hline Composition & 30.84 & 32.22 & 34.35 & 33.69 & 43.09 & 25.32 & 32.12 & 55.92 & 56.36 & 46.28 & 32.84 & 54.79 \\
\hline
\end{tabular}


Table 2. Major oxide and trace element deteminations and CIPW normative mineralogy for selected igneous rocks from Sleetmute C-7, C-8, D-7. and D-8 Quadrangles - Continued - page 5 of 5

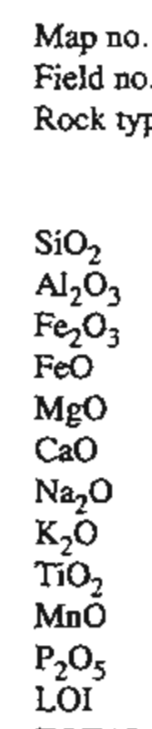

TOTAL

$\begin{array}{lr}\mathrm{Cr} & <1 \\ \mathrm{Rb} & \mathrm{I} \\ \mathrm{Sr} & 142 \\ \mathrm{Y} & <1 \\ \mathrm{Zr} & 139 \\ \mathrm{Nb} & <10 \\ \mathrm{Ba} & 3.880\end{array}$

Quartz

Corundum

Orhoclase

Antice

Anorthice

Olivine

Hyperstene

Magnetile

Imenite

Apatite

Differentation

Index

Plagioclase

Composition

\begin{tabular}{|c|c|c|}
\hline 49 & 50 & 51 \\
\hline $91 \mathrm{BT} 13^{\mathrm{a}}$ & 91BT9a & $91 \mathrm{BT} 63^{\mathrm{a}}$ \\
\hline Felsite & Granite & Granite \\
\hline (TKgp) & Porphyry & Porphyry \\
\hline & (TK $\mathrm{PD})$ & (TKp) \\
\hline 72.60 & 72.70 & 63.20 \\
\hline 14.20 & 14.50 & 15.90 \\
\hline 1.06 & 1.43 & 2.40 \\
\hline 0.90 & 0.50 & 3.50 \\
\hline 0.63 & 0.46 & 1.48 \\
\hline 0.27 & 1.03 & 3.28 \\
\hline 2.35 & 2.77 & 3.93 \\
\hline 5.02 & 4.07 & 3.41 \\
\hline 0.25 & 0.25 & 0.91 \\
\hline 0.05 & 0.02 & 0.10 \\
\hline 0.13 & 0.12 & 0.24 \\
\hline 2.00 & 2.00 & 1.47 \\
\hline 99.46 & 99.85 & 99.82 \\
\hline
\end{tabular}

\begin{tabular}{c}
52 \\
$91 \mathrm{BT} 50 \mathrm{a}^{8}$ \\
Gramite \\
Porphyry \\
(TK.0p) \\
\hline 73.60 \\
15.00 \\
0.53 \\
0.60 \\
0.13 \\
1.00 \\
3.97 \\
4.26 \\
0.05 \\
0.02 \\
0.08 \\
0.93 \\
\hline 100.17
\end{tabular}

53
$91 B T 50 b^{2}$

9IBTSOba
Granite

$\begin{array}{ccc}54 & 55 & 56 \\ 91 \mathrm{BT} 50^{\mathrm{a}} & 91 \mathrm{~B} T 51^{\mathrm{a}} & 91 \mathrm{BT} 54^{\mathrm{a}}\end{array}$

Granite Granite

(TKgr) Cokgp) Th

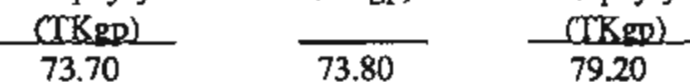

72.20
14.40

73.70
15.00

$15.20 \quad 11.90$

$\begin{array}{rrr}0.54 & 0.42 & 0.38 \\ 0.60 & 0.10 & 0.40\end{array}$

$\begin{array}{lll}0.64 & 0.10 & 0.40 \\ 0.13 & 0.14 & 0.11\end{array}$

$\begin{array}{lll}0.99 & 0.14 & 0.11 \\ 4.03 & 4.25 & 0.81\end{array}$

$\begin{array}{lll}4.03 & 4.25 & 3.18 \\ 4.33 & 4.27 & 3.40 \\ 0 & 0.05 & 0.04\end{array}$

$\begin{array}{lll}0.05 & 0.03 & 0.04 \\ 0.02 & 0.02 & 0.01 \\ 0.08 & 0.06 & 0.06\end{array}$

\begin{tabular}{rrr}
0.02 & 0.02 & 0.01 \\
0.08 & 0.06 & 0.06 \\
0.77 & $\frac{1.54}{100.31}$ & $\frac{1.00}{100.49}$ \\
\hline 100.24 & &
\end{tabular}

Trace Elements (in ppm)

$\begin{array}{rrr}<10 & <10 & 26 \\ 157 & 129 & 119 \\ 142 & 239 & 276 \\ <10 & 12 & 37 \\ 139 & 134 & 331 \\ <10 & 24 & 19 \\ 180 & 2390 & 1.450\end{array}$

$\begin{array}{rr}13 & <10 \\ 177 & 138 \\ 100 & 189 \\ <10 & 34 \\ 55 & 220 \\ 26 & 13 \\ 574 & 1180\end{array}$

$<10$
183
89
$<10$
66
31
604

$\begin{array}{rr}<10 & <10 \\ 221 & 135 \\ 80 & 76 \\ <10 & <10 \\ 42 & 44 \\ 22 & 13 \\ 427 & 615\end{array}$

CIPW Norms (weight/percent)

\begin{tabular}{rrr}
39.36 & 39.33 & 17.53 \\
4.85 & 4.04 & 0.36 \\
30.47 & 24.59 & 20.57 \\
20.42 & 23.97 & 33.94 \\
0.50 & 4.42 & 15.01 \\
0.00 & 0.00 & 0.00 \\
0.00 & 0.00 & 0.00 \\
2.17 & 1.17 & 7.28 \\
1.43 & 1.71 & 2.99 \\
0.49 & 0.49 & 1.76 \\
0.31 & $\underline{0.28}$ & 0.57 \\
\hline 100.00 & 100.00 & 100.01
\end{tabular}

3206

$\begin{array}{rr}2.25 & 0.00 \\ 25.38 & 25.78\end{array}$

$33.87 \quad 39.13$

4.48
0.00 $\quad 5.86$

$\begin{array}{ll}0.00 & 0.06 \\ 0.00 & 0.00\end{array}$

$\begin{array}{ll}0.00 & 0.00 \\ 1.01 & 1.82 \\ 0.67 & 1.31\end{array}$

$0.67 \quad 1.31$

$\begin{array}{ll}0.10 & 0.40 \\ 0.19 & 0.16\end{array}$

$\frac{0.19}{100.01} \quad \frac{0.16}{100.01}$

91.31

90.40

$31.49 \quad 32.07$

$2.09-2.89$

$\begin{array}{lll}25.74 & 25.55 & 20.20 \\ 34.30 & 36.4 ! & 27.06\end{array}$

$\begin{array}{lll}4.42 & 2.02 & 3.06 \\ 0.00 & 0.65\end{array}$

$\begin{array}{lll}0.00 & 0.00 & 0.00 \\ 0.00 & 0.00 & 0.00\end{array}$

$\begin{array}{lll}0.00 & 0.00 & 0.00 \\ 1.00 & 0.35 & 0.68\end{array}$

$\begin{array}{lll}1.00 & 0.35 & 0.68 \\ 0.69 & 0.51 & 0.50\end{array}$

$\begin{array}{rrr}0.10 & 0.06 & 0.08\end{array}$

\begin{tabular}{lll}
0.10 & 0.06 & 0.08 \\
0.19 & 0.14 & 0.14 \\
\hline
\end{tabular}

$\begin{array}{lll}90.25 & 87.89 & 72.04\end{array}$

$\begin{array}{lll}0.98 & 15.57 & 30.66\end{array}$

11.68

13.03

91.53

94.03

93.29

$\begin{array}{lll}11.42 & 5.26 & 11.89\end{array}$ 